\title{
La politique agricole suisse entre économie, écologie et société
}

\section{Dominique Barjolle}

\section{(2) OpenEdition}

1 Journals

\section{Édition électronique}

URL : http://journals.openedition.org/economierurale/2510

DOI : $10.4000 /$ economierurale. 2510

ISSN : 2105-2581

\section{Éditeur}

Société Française d'Économie Rurale (SFER)

\section{Édition imprimée}

Date de publication : 1 février 2010

Pagination : 6-8

ISSN : 0013-0559

\section{Référence électronique}

Dominique Barjolle, «La politique agricole suisse entre économie, écologie et société », Économie rurale [En ligne], 315 | Janvier-février 2010, mis en ligne le 05 février 2012, consulté le 19 avril 2019. URL : http://journals.openedition.org/economierurale/2510 ; DOI : 10.4000/economierurale.2510 


\title{
Introduction \\ LA POLITIQUE AGRICOLE SUISSE ENTRE ÉCONOMIE, ÉCOLOGIE ET SOCIÉTÉ
}

\author{
Dominique BARJOLLE \\ AGRIDEA \\ Développement de l'agriculture et de l'espace rural \\ Lausanne
}

\begin{abstract}
e dossier explore quelques aspects de la politique agricole et de l'économie rurale suisses. Fondée sur la notion de sécurité alimentaire dans l'après-guerre, la politique agricole suisse a d'abord été marquée par la volonté de préserver une capacité productive capable de nourrir sa population en cas de crise ou de conflit. Elle a donc établi une forte protection aux frontières et des aides sélectives toujours plus importantes en faveur des exploitations situées en zone défavorisée, particulièrement la montagne qui regroupe $70 \%$ des zones agricoles.
\end{abstract}

Face aux excédents coûteux de la production laitière et sous la pression de vives critiques des dégâts collatéraux de l'intensification sur les ressources naturelles, la politique agricole suisse a complètement changé de paradigme dans la conception de ses outils ${ }^{1}$. Entre 1992 et 2009, les proportions des montants consacrés aux aides au marché et aux compensations de prestations particulières pour la protection des ressources naturelles ont été inversées. Ce dossier s'ouvre sur l'article de Jean-Christophe Kroll, Dominique Barjolle et Marjorie Jouen qui expliquent et chiffrent ce mouvement. Ce faisant, le principe de base de l'intervention publique dans le secteur agricole est actuellement de compenser le manque à gagner économique entre une production intensive ne faisant aucun cas de la protection des ressources naturelles et une production agricole durable fondée sur un modèle d'exploitation familiale. Ce manque à gagner consiste en un système élaboré de paiements directs ciblés et systématiques.

En cela, la Suisse affiche un choix de société, et elle le fait d'autant plus qu'a été adopté lors d'un vote populaire en juin 1996, une base constitutionnelle qui fonde le choix des outils et ancre les soutiens publics dans le long terme. Marguerite Paus et Sophie Réviron y font référence dans une annexe de leur article ${ }^{2}$. Pour autant, les débats actuels font rage entre la profession et le gouvernement et divisent même la profession. En effet, le mouvement de globalisation des échanges n'épargne pas la Suisse, qui est aujourd'hui confrontée à une ouverture imminente de ses frontières dans le cadre des accords agricoles l'Organisation mondiale du commerce (OMC). Pour adapter sa production rapidement et inventer un nouvel équilibre entre la production durable et des prix potentiellement en forte baisse, le gouvernement a entrepris en 2008 deux chantiers importants :

- une refonte totale du système des paiements directs,

- la libéralisation totale des échanges avec l'Union européenne.

1. Pour une revue complète, le lecteur pourra se référer à l'ouvrage L'agriculture suisse dans son nouveau rôle, Barjolle et al. (2008), Presses polytechniques universitaires romandes.

2. $C f$. l'annexe en page 44 .

6 • ÉCONOMie RuRALe 315/JANVIER-FÉVRIER 2010 
Le choix d'une intégration forte dans le marché européen permettra de positionner l'agriculture suisse au sein d'un espace concurrentiel aux conditions pédoclimatiques comparables pour y trouver des avantages comparatifs qui permettent la survie pour les exploitations familiales.

Ce pari est contesté par la profession elle-même, confrontée de plein fouet aux conséquences très dures de la suppression des quotas laitiers en 2009, en pleine crise de surproduction laitière à l'échelle européenne. Les baisses très fortes des prix du lait (-30\% entre septembre 2008 et mai 2009) et des céréales (-20\% entre 2008 et 2009) interrogent les producteurs, et surtout les plus intensifs, sur l'avenir d'un modèle agricole qui cherche à concilier une production compétitive, durable et avec une évolution des structures socialement supportable.

Cette politique agricole singulière, qui peut perdurer du fait de l'isolement de la Suisse en-dehors de l'Union européenne (UE), a des effets positifs indéniables en termes de protection des ressources naturelles, et notamment des paysages. Ceci peut s'expliquer aisément par l'importance des montants des paiements directs que l'on pourra lire dans l'article de Jean-Christophe Kroll, Dominique Barjolle et Marjorie Jouen (op. cit.). Alors que l'article de Valérie Miéville-Ott et Yvan Droz permet de situer toute la problématique de la protection paysagère et des modalités de sa conception en Suisse. La conception du lien avec la politique agricole est que «les paiements directs permettent de freiner la diminution des exploitations agricoles et contribuent ainsi au maintien d'un paysage ouvert par la simple préservation de l'activité agricole. Le qualificatif d'ouvert n'est précisé d'aucune manière. Des prestations paysagères plus spécifiques peuvent être également rémunérées, telles les surfaces de compensation écologique que l'agriculteur doit mettre à disposition s'il veut toucher les paiements directs. Là encore, une analogie est posée entre la qualité écologique et la qualité paysagère : plus un paysage est diversifié écologiquement parlant, plus il est censé être apprécié sur le plan esthétique. » Toute la discussion est cependant de savoir si cette politique, décrétée comme une politique unique, prend en compte les demandes sociales locales en raison de l'appréciation subjective de l'esthétisme. Le système actuel des paiements directs ne permet pas d'influencer la qualité paysagère, mais la réforme mise en discussion actuellement s'oriente dans cette direction.

Dans la perspective de la libéralisation complète des marchés agricoles avec l'UE, la question des signes de qualité se pose avec acuité. En effet, la stratégie encouragée par la politique agricole est celle de la différenciation par les qualités particulières, liées à l'origine ou à un mode de production spécifique. Cette question est abordée dans l'article de Marguerite Paus et Sophie Réviron (op. cit.), sous l'angle de l'impact des stratégies de différenciation sur le développement durable au niveau local. Les stratégies régionales de différenciation des produits, appuyées fermement par des politiques agricoles des Etats cantonaux (la Suisse est un Etat fédéral de 26 cantons), ont des impacts territorialisés intéressants. Cependant, d'autres travaux dont la revue bibliographique est faite en première partie de l'article, ont démontré clairement qu'ils ne sont pas systématiques mais qu'ils dépendent de nombreux autres facteurs tels que la capacité stratégique des acteurs, le soutien des mesures d'appui et les synergies avec la promotion d'autres activités régionales telles que le tourisme. 
L'articulation des politiques agricoles et de développement rural est l'un de ces facteurs. Le grand chantier qui attend les générations futures de politiciens, qu'ils soient Suisses ou Européens sera de consolider une politique de développement mariant localement le rural et l'agricole, associant la politique agricole sectorielle avec les politiques transversales de l'aménagement du territoire et du développement territorial plurisectoriel. Les acteurs ont besoin de s'approprier les instruments politiques pour les combiner selon des stratégies adaptées aux défis à relever dans leurs régions spécifiques.

Les alliés naturels de la Suisse en matière de politique agricole sont les pays de l'arc alpin, Autriche, Slovénie, mais aussi les régions de montagne d'Italie (Aoste, Trentin), de France (Provence-Rhône-Alpes-Côte d'Azur, Centre et Pyrénées), d'Allemagne (Baden-Würtenberg, Bavière), et des pays de la chaîne des Carpates (Bulgarie, Roumanie) pourraient s'y rallier après la phase de transition qui les caractérise pour l'instant. Ces pays et régions ont la vision d'une agriculture fondée sur l'exploitation familiale, la multifonctionnalité et la mise en valeur agricole de toutes les zones, y compris défavorisées notamment les espaces alpins, dans le but d'un paysage cultivé, de zones aux ressources préservées entretenues par l'agriculture. Cette analyse a été également faite par Perrot et $a l .^{3}$ dans une étude récente des productions laitières dans les zones de montagne européennes.

Cette vision de l'agriculture peut-elle survivre en Suisse comme au sein de l'Union européenne ? Une régionalisation plus marquée de la politique agricole européenne sera nécessaire pour y parvenir. Il convient de relire la conclusion de l'article de Jean-Christophe Kroll, Dominique Barjolle et Marjorie Jouen (dans ce numéro) : «Dans cette logique, la politique agricole suisse n'apparaîtrait plus comme un vestige du passé, condamné par l'OMC, mais plutôt comme une préfiguration des politiques que certaines régions riches de l'Europe seraient en situation de développer dans le cadre d'un recentrage de la PAC sur son second pilier. On évoluerait alors vers une situation paradoxale où la "multifonctionnalité » de l'agriculture passerait par une spécialisation fonctionnelle des espaces, producteurs de marchandises pour les uns, et de services environnementaux ou récréatifs pour les autres».

3. Perrot C., Derville M., Monniot C., Richard M. (2009). Le lait dans les montagnes européennes. Un symbole menacé. Communication aux $3 \mathrm{R}$, Institut de l'élevage 